\title{
Effect of Trichinella spiralis intervention on Citrobacter rodentium-induced experimental colitis in mice
}

\author{
Gui-Lian Yang ${ }^{1}$ \\ ${ }^{1}$ Jilin Agricultural University
}

July 27, 2020

\begin{abstract}
Materials and Methods: In the present study, 36 male BALB/c mice aged 6-8 weeks were randomly divided into four groups: the control group (PBS), the T. spiralis infection group (TS), the C. rodentium-induced colon inflammation model group (CR) and the T. spiralis pre-infected and C. rodentium induced colitis group (TS+CR). The mice were sacrificed 7 and 14 days after the establishment of the model. The changes in various colitis indicators were used to investigate the effect of T. spiralis infection on the C. rodentium-induced mouse CD model. Results: The results showed that the weight, DAI score, and macroscopic and microscopic colon damage in the TS+CR group was significantly decreased compared with that observed in the CR group. Flow cytometry results revealed that T. spiralis infection could reduce the expression of IFN- $\gamma$, IL-17 and IL-12 and increase that of IL-4 and IL-10 in colitis mice. Compared with that observed in the control group, the number of CD4+CD25+Foxp3+ regulatory $\mathrm{T}$ cells (Tregs) in the spleens and mesenteric lymph nodes (MLNs) of mice in the TS group was increased $(\mathrm{P}<0.01)$. Compared with that observed in the TS group mice, the expression of CD4+CD25+Foxp3+Tregs in the spleens and MLNs of the $\mathrm{TS}+\mathrm{CR}$ group mice was decreased $(\mathrm{P}<0.05)$. ELISA results revealed that compared with the control group, the TS group produced a strong IgG1 response $(\mathrm{P}<0.001)$ and low level of IgG2a response $(\mathrm{P}>0.05)$, while increased expression of IgG2a in serum was detected in the $\mathrm{CR}$ group mice $(\mathrm{P}<0.05)$.
\end{abstract}

Effect of Trichinella spiralis intervention on Citrobacter rodentium-induced experimental colitis in mice

Ying Xue ${ }^{1,2,3}$, Yun-Fei Xu ${ }^{1,2,3}$, Tian-Xu Pan ${ }^{1,2,3}$, Hai-Bin Huang ${ }^{1,2,3}$, Jun-Yi Li ${ }^{1,2,3}$, Bo Zhang ${ }^{1,2,3}$, Yue Tang $^{1,2,3}$, Chun-Wei Shi ${ }^{1,2,3}$, Gui-Lian Yang ${ }^{1,2,3 *}$, Chun-Feng Wang ${ }^{1,2,3 *}$

1.College of Animal Science and Technology, College of Veterinary Medicine, Jilin Agricultural University, Changchun, China.

2.Jilin Provincial Engineering Research Center of Animal Probiotics, Jilin Agricultural University, Changchun, China.

3.Key Laboratory of animal production and product quality safety of Ministry of Education, Jilin Agricultural University, Changchun, China.

*Corresponding authors:

Gui-Lian Yang, College of Animal Science and Technology, College of Veterinary Medicine, Jilin Provincial Engineering Research Center of Animal Probiotics, Jilin Agricultural University, 2888 Xincheng Street, Changchun 130118, China. E-mail: yangguilian@jlau.edu.cn, Tel./fax: +86 43184533425.

Chun-Feng Wang, College of Animal Science and Technology, College of Veterinary Medicine, Jilin Provincial Engineering Research Center of Animal Probiotics, Jilin Agricultural University, 2888 Xincheng Street, Changchun 130118, China. E-mail: wangchunfeng@jlau.edu.cn, Tel./fax: +86 43184533426. 


\section{List of abbreviations}

IBD: inflammatory bowel disease

T. spiralis : Trichinella spiralis

C. rodentium : Citrobacter rodentium

Tregs: regulatory $\mathrm{T}$ cells

MLNs: mesenteric lymph nodes

UC: ulcerative colitis

CD: Crohn's disease

EPEC: Escherichia coli

EHEC: enterohaemorrhagic E. coli

TGF- $\beta$ : Transforming growth factor- $\beta$

IL: interleukin

ELISA: Enzyme-linked immunosorbent assay

SPF: specific pathogen free

DAI: disease activity index

TNBS: 2,4,6-Trinitrobenzene sulfonic acid

DNBS: dinitrobenzenesulfonate

\section{Summary}

Objectives: Epidemiological surveys have shown that the incidence of inflammatory bowel disease (IBD) is relatively high in developed countries but relatively low in countries with poor sanitation and dense populations. Good hygiene and low parasitic infections increase the susceptibility of individuals to IBD. The goal of the present study was to assess the immune characteristics of hosts infected with Trichinella spiralis(T. spiralis ) and the relationship between intestinal worms and IBD. The ability of T. spiralis infection to ameliorate the effects of Citrobacter rodentium ( $C$. rodentium ) in a mouse IBD animal model was assessed, and the possible immunological mechanism associated with this prevention and treatment of IBD was evaluated.

Materials and Methods: In the present study, 36 male BALB/c mice aged 6-8 weeks were randomly divided into four groups: the control group (PBS), the T. spiralis infection group (TS), the C. rodentium -induced colon inflammation model group (CR) and the T. spiralis pre-infected and C. rodentium induced colitis group (TS+CR). The mice were sacrificed 7 and 14 days after the establishment of the model. The changes in various colitis indicators were used to investigate the effect of $T$. spiralis infection on the $C$. rodentium -induced mouse CD model.

Results: The results showed that the weight, DAI score, and macroscopic and microscopic colon damage in the TS+CR group was significantly decreased compared with that observed in the CR group. Flow cytometry results revealed that $T$. spiralis infection could reduce the expression of IFN- $\gamma$, IL-17 and IL-12 and increase that of IL-4 and IL-10 in colitis mice. Compared with that observed in the control group, the number of CD $4^{+} \mathrm{CD} 25^{+} \mathrm{Foxp}^{+}{ }^{+}$regulatory T cells (Tregs) in the spleens and mesenteric lymph nodes (MLNs) of mice in the TS group was increased $(P<0.01)$. Compared with that observed in the TS group mice, the expression of CD $4^{+} \mathrm{CD} 25^{+}$Foxp $3^{+}$Tregs in the spleens and MLNs of the TS+CR group mice was decreased $(P<0.05)$. ELISA results revealed that compared with the control group, the TS group produced a strong 
IgG1 response $(P<0.001)$ and low level of IgG2a response $(P>0.05)$, while increased expression of IgG2a in serum was detected in the $\mathrm{CR}$ group mice $(P<0.05)$.

Conclusion: The results of the present study demonstrated that T. spiralis -infected mice induced balanced Th2 immune responses that balanced Th1 immune responses stimulated by $C$. rodentium to ameliorate intestinal inflammation.

Key words: Trichinella spiralis ; Citrobacter rodentium ; inflammatory bowel disease

\section{Introduction}

IBD is a chronic intestinal disorder that primarily manifests as a specific immune response of the mucosal immune system towards intestinal pathogenic microorganisms. Human IBD can be divided into two types: ulcerative colitis (UC) and Crohn's disease (CD) [1]. UC typically involves Th2- and Th17-type inflammation, and the superficial portion of the colon is prone to ulcers. CD is primarily based on Th1-type inflammation, and the lesions are typically transmural and may spread throughout the intestine [2]. Compared to developing countries, the incidence of inflammatory bowel disease in developed Western countries is relatively high. This phenomenon can be explained by the "hygiene hypothesis", which describes how improved sanitary conditions reduce the chances individuals will come into contact with pathogenic organisms, increasing the incidence of autoimmune and inflammatory diseases [3-5]. Antiparasitic drugs and good hygiene practices in developed countries have effectively controlled parasitic infections, but with the elimination of parasitic infections, the incidence of immune diseases, including inflammatory bowel disease, has also increased [6].

As a non-invasive organism, C. rodentium uses attaching and effacing (A/E) lesion formation as its major tissue-targeting mechanism, resulting in infection. This approach is consistent with the mechanisms observed in other enteropathogenic pathogens, such asEscherichia coli (EPEC) and enterohaemorrhagic E. coli (EHEC). As a natural mouse pathogen, C. rodentium provides an excellent in vivo model to study A/E lesion-forming pathogens, including EPEC and EHEC, because both EHEC and EPEC are poorly pathogenic in mice. However, infection with $C$. rodentium has also been investigated as a model for studying IBD, because the colonic pathology of the mouse shares several similarities with clinical IBD [7]. In addition, this model provides potential mechanisms for increasing the understanding of how host immunity that plays a role in limiting the infection of the mucosal epithelium of the colon and clearing pathogens [8].

Parasitic infection can induce an immune response in the host, causing polarization towards a Th2-type response, which is characterized by the expression of the Th2-type cytokines IL-4, IL-5 and IL-13 [9]. Parasites downregulate $\mathrm{T}$ and $\mathrm{B}$ cell responses with respect to Tregs, anti-inflammatory cytokines IL-10 and transforming growth factor TGF- $\beta$ and regulates the Th1- and Th2-type immune responses [10]. Parasites can establish a parasitic lifestyle by regulating the host immune response, which simultaneously protects the host from excessive inflammation that causes damage to tissues and organs [11]. In recent years, experimental and clinical studies have shown that parasitic infections can protect the host from inflammatory bowel disease. For example, the intestinal parasites T. spiralis, Swine whipworm (Trichuris muris ) and Hymenolepis diminuta can provide protection to IBD in mice $[12,13]$. Similarly, UC and CD symptoms in IBD patients infected with T. muris are alleviated [14]. The mechanism of intestinal parasite treatment of IBD is not yet clear, and many aspects of this process remain to be elucidated. In the present study, we constructed an experimental mouse enteritis model induced by $C$. rodentium, studied the immune system effects of T. spiralis treatment on inflammatory bowel disease in mice, and elucidated the effects of T. spiralis intervention in IBD at the molecular and cellular levels to identify the potential immunological mechanisms involved to provide a theoretical basis for clinical treatment of human IBD.

\section{Materials and methods}

Parasite isolate, storage and recovery of $C$. rodentium, animals, culture medium and growth conditions

The T. spiralis isolate ISS534 used in the present study was obtained from domestic pigs in Henan Province, China, and maintained in Kunming mice in the Jilin Provincial Engineering Research Center of Animal 
Probiotics. C. rodentium ATCC 51459 was provided by Dr Haining Shi (Harvard Medical School, Boston, MA, USA) and cultured in Luria-Bertani medium at $37^{\circ} \mathrm{C}$ with shaking. Male BALB/c mice (specific pathogen free, SPF) aged 6-8 weeks were purchased from Beijing Hua fu kang Biotechnology. The study protocol was approved by the Jilin Agriculture University Veterinary Research Ethics Committee, and all procedures were performed in strict accordance with the guidelines of the Chinese National Institute of Health Guide for the Care and Use of Laboratory Animals. Care was taken to minimize the number of animals used and to reduce their suffering. Before the experiment, the mice were allowed to eat and drink freely for at least 4 days to adapt to the feeding environment. Throughout the experiment, the mice were allowed to eat and drink freely, and the bedding materials were changed regularly to ensure an excellent feeding environment.

\section{Experimental grouping}

The experimental mice were randomly divided into four groups, with more than nine mice in each group: the control group (PBS), the T. spiralis -infected group (TS), the C. rodentium -induced colitis model group (CR), and the T. spiralis -pre-infected C. rodentium -induced colitis group (TS+CR). At the beginning of the experiment, each mouse in the TS and TS+CR groups was orally infected with 400 individual T. spiralis muscle larvae. After 21 days of infection, the control and TS groups were administered PBS, and the CR and TS+CR groups were administered $2 \times 10^{9} \mathrm{CFU} / \mathrm{mLC}$. rodentium. Subsequently, the faeces of mice in the $\mathrm{CR}$ and TS+CR groups were collected every day from days 1 to 14 post C. rodentium infection, the colony count of faeces was determined, and the number of $C$. rodentium per gram of faeces $(\mathrm{CFU} / \mathrm{g})$ was calculated. The mice were sacrificed under euthanasia on days 7 and 14, and the weights of the mice in the four groups was determined daily.

\section{Collection and oral administration of $T$. spiralis larvae in mouse muscles}

T. spiralis were maintained in our laboratory through serial infection of rats. The parasites were isolated from the infected mice through dissection, were their muscle tissues were harvested and bones were removed. The muscles were treated with an artificial digestion solution (1\% pepsin and $1 \%$ concentrated hydrochloric acid) for $4 \mathrm{~h}$ at $37^{\circ} \mathrm{C}$. Subsequently, the mixture was filtered using an 80-mesh filter and rinsed thrice. The larvae were subsequently harvested in accordance with the modified Bellman's method. Then, the vitality of the larvae was assessed, and they were enumerated under a microscope. The mice in the TS and TS+CR groups were orally infected with 400 individual larvae.

\section{C. rodentium-induced colitis}

Jiang et al. (2016) established the $C$. rodentium -induced colitis model approach [15] that was used the present study. The mice in the CR and TS+CR groups were rapidly administered $2 \times 10^{9} \mathrm{CFU} / \mathrm{mL}$ C. rodentium 24 $\mathrm{h}$ after fasting, while the mice in the control and TS groups were administered the same amount of PBS. The colitis mouse model elicited a Th1-type immune response, which was similar to that observed in human CD. Inflammation was assessed based on bellowed parameters, including clinical disease activity and the macroscopic and microscopic inflammation scores in the colon tissue. The grading was performed by three investigators blinded for the treatment of the mice.

\section{T. spiralis infection}

After the mice were sacrificed, their diaphragms were examined under a microscope to assess the success of T. spiralis infection, and the success rates of infection and parasitic density were calculated.

\section{Disease activity index evaluation}

The mice were observed daily in terms of the changes in their mental status, activity, hair gloss, appetite, and defecation (presence of blood, stool pattern, defecation frequency). Each group of mice was scored for the disease activity index (DAI) according to international standards: DAI = (weight loss score + stool score + blood stool score) $/ 3$. To detect faecal occult blood, we used the benzidine method.

\section{Macroscopic and microscopic assessment of colon injury}


After the mice were sacrificed by cervical dislocation, the abdominal wall was opened, and the intestine was exposed. Then, the entire segment of the colon from the rectum to the cecocolic junction was removed, opened and rinsed thoroughly with normal saline, after which the isolated colon was examined for macroscopic damage. Scores were assessed by using the following damage scoring system [16]: 0: no damage; 1: localized hyperaemia without ulcers; 2: linear ulcers with no significant inflammation; 3: linear ulcer with inflammation at one site; 4: ulcer and inflammation at two or more locations; and 5: two or more major sites of inflammation and ulceration or one major site of inflammation and ulceration extending more than $1 \mathrm{~cm}$ along the colon. Then, colon specimens were fixed in $10 \%$ paraformaldehyde for hour(s), after which paraffin sections were generated and haematoxylin and eosin $(\mathrm{H} \& \mathrm{E})$ staining was performed. The pathological sections of the colon were observed under an optical microscope (Leica, GER). Histological damage was assessed using the criteria of Wallace and Keenan [17]: 0: intact tissue construction with no apparent damage; 1: damage limited to surface epithelium; 2: localized ulcer confined to mucosa; 3: focal, transmural inflammation and ulceration; 4: extensive transmural ulceration and inflammation adjacent to normal mucosa; and 5: extensive transmural ulceration and inflammation involving entire section. All data were obtained from three separate experiments, and the scores were assigned by three observers without knowing the state of the mice (Table 1).

\section{Detection of IgG1 and IgG2a in serum by ELISA}

Serum samples were collected 14 days after $C$. rodentiuminfection. The changes in IgG1 and IgG2a levels in serum were assessed using an ELISA kit (Bioss, CHN) following the manufacturer's instructions for cytokine detection. Cytokine levels were expressed per milligram of total protein. All data were obtained from three separate experiments.

\section{Detection of $\mathrm{CD}^{+}{ }^{+} \mathrm{CD}^{2} 5^{+}{ }^{\text {Foxp }}{ }^{+}$Tregs and Th cells in spleens and MLNs by flow cytometry}

Spleen and MLN lymphocyte single cell suspensions were prepared, and $1 \times 10^{6}$ cells were added to each tube. The cells were resuspended in PBS and incubated for $30 \mathrm{~min}$ in the dark with the following antibody combinations: FITC-rat-anti-mouse CD4, PE-rat-anti-mouse Foxp3 and APC-rat-anti-mouse CD25; FITCrat-anti-mouse IL-17 and PE-Rat-anti-mouse IL-12; FITC-rat-anti-mouse IL-10 and PE-rat-anti-mouse IFN$\gamma \cdot$ or PE-rat-anti-mouse IL-4 (all antibodies were obtained from BD, USA). The cells were washed and resuspended again for flow cytometry analysis. All data were obtained from three separate experiments.

\section{Statistical analysis}

All results are presented as the means \pm standard error. Data were evaluated using one-way ANOVA with SPSS 13.0. Differences were considered significant at $P<0.05$. All statistical analyses were performed using GraphPad Prism 7.

\section{Results}

\section{Survival rate}

The survival rate of mice in the CR group was only $65.78 \%$, significantly lower than that observed for the TS + CR group $(89.78 \% ; P<0.05)$, while the survival rate of mice in the control and TS groups was $100 \%$.

\section{Weight change}

The day the $C$. rodentium model establishment was experimental day 0 . The body weights of mice in each group were recorded daily from the beginning until the end of the experiment, and the results are presented in Fig. 1. The weights of mice in the CR group were significantly lower those of the control group mice. The weights of mice in the CR group decreased by $6.62 \%$ on day 7 before gradually increasing by $0.76 \%$ on day 14. The weights of mice in the TS+CR group decreased relatively slowly compared to that observed in the CR group, decreasing by $2.22 \%$ on day seven to the lowest value observed before gradually increasing by $8 \%$ on day 14 .

\section{Changes in the amount of $C$. rodentium in faeces}


From day 1 to 14 post $C$. rodentium infection, the excretion of $C$. rodentium in the faeces of the TS+CR group was lower than that observed in the CR group. From day 1 to 14 post $C$. rodentiuminfection, the $C$. rodentium excretion by the TS+CR group was significantly reduced, that observed in the CR group remained at a high level (Fig. 2).

\section{DAI score}

Twenty-four hours after the $C$. rodentium model was established, mice in the control colitis group manifested lethargy, sluggishness, appetite loss, rough hair, diarrhoea, bloody stools or faecal occult blood $(+++)$, and weight loss. These symptoms were most evident 3 days after the start of modelling. All the symptoms of mice of the TS+CR group were less severe than those observed in the CR group and were significantly different on days 1 and $3(P<0.05)$. The conditions of the surviving mice in both groups started to gradually improve on day 4 , and the mice were relieved on day 7 . At this time, in contrast to the mice in the CR group, the mice in the TS+CR group were still recovering. The DAI scores of the three groups at different time points are shown in Fig. 3.

\section{Colon pathological changes}

To further evaluate the protective effects of T. spiralis in C. rodentium infection, on day 14 after the start of modelling, distal colon samples were collected and evaluated by H\&E staining. As expected, no pathogenic changes were observed in either the control group or TS groups (Fig. 4AC), whereas the CR group exhibited typical pathological changes, including colonic epithelial cells proliferation, intestinal wall oedema, large amounts of inflammatory cell infiltration in the lamina propria, elongated crypts and the reduction or disappearance goblet cells (Fig. 4B). In the TS+CR group, the hyperplasia of colonic epithelial tissue disappeared, the lesions were limited to the mucosa, and the lamina propria of the mucosa was scattered with inflammatory cells ((Fig. 4D).

\section{Changes in IgG1 and IgG2a levels in serum}

After 14 days of $C$. rodentium infection, serum samples were collected, and ELISA was used to assess the changes in IgG1 and IgG2a levels in mouse serum. Compared with the control group, the TS group produced a strong IgG1 response $(P<0.001)$ and a low-level IgG2a response $(P>0.05)$, with an increased expression of IgG2a observed in serum from CR group mice $(P<0.05)$. This result showed that $C$. rodentiuminfection primarily induces a Th1-type immune response in mice, while $T$. spiralis infection tended to induce a Th2type immune response. Compared with the CR group, the TS+CR group had increased IgG1 expression $(P$ $<0.01)$ and decreased IgG2a expression $(P<0.01)$, while compared with the TS group, the TS+CR group exhibited increased IgG2a expression $(P<0.01)$. These results showed that the Th2 type immune response induced by T. spiralis inhibits the Th1 immune response caused by C. rodentium infection (Fig. 5).

\section{Changes in Th cells and $\mathrm{CD}^{+}{ }^{+} \mathrm{CD}^{2} 5^{+} \mathrm{Foxp}^{+}{ }^{+}$Tregs in the spleens and MLNs of mice}

The mice were sacrificed 7 and 14 days after the start of $C$. rodentium modelling. The spleens and MLNs of each group were removed, and the number of Th cells was assessed by flow cytometry. The results showed that 7 and 14 days after $C$. rodentium infection, the expression of IFN- $\gamma$ and IL-12 in the spleens and MLNs of mice in the $\mathrm{CR}$ group increased $(P<0.01)$. Compared with that observed in the CR group, the expression of IFN- $\gamma$ and IL-12 in the spleens and MLNs of mice the TS group was decreased $(P<0.01)$, and while that of IL-10 and IL-4 was increased $(P<0.05)$. These results showed that $C$. rodentium primarily induced the production of Th1 cytokines in mice, while T. spiralis infection primarily induced the production of Th2 cytokines. Compared with that observed in the CR group, the expression of IFN- $\gamma$ and IL-12 in the spleens and MLN of mice in the TS+CR group was reduced $(P>0.05)$, showing that the Th2-type cytokine response caused by $T$. spiralis infection could inhibit the development of Th1-type inflammation induced by $C$. rodentium . Similarly, the expression of IL-10, IL-4 and IL-17 in the spleens and MLNs of mice in the TS+CR group was significantly lower than that observed in the TS group $(P<0.05)$. These results successfully showed that the Th1-type cytokine response caused by $C$. rodentium infection can inhibit the Th2-type immune response induced by T. spiralis (Fig. 6, 7). 
The mice were sacrificed 14days after $C$. rodentium modelling. The spleen and MLN of each group were removed, and the expression of $\mathrm{CD} 4{ }^{+} \mathrm{CD} 25^{+} \mathrm{Foxp} 3^{+}$Tregs was detected by flow cytometry. The result showed that compared with that in the control group, the number of $\mathrm{CD} 4{ }^{+} \mathrm{CD} 25^{+} \mathrm{Foxp} 3^{+}$Tregs in the spleens and MLNs of mice in the TS group was increased $(P<0.01)$, showing that $T$. spiralis infection could induce $\mathrm{CD} 4{ }^{+} \mathrm{CD} 25^{+}$Foxp $3^{+}$Treg production in mice. Compared with that observed in the TS group mice, the number of $\mathrm{CD} 4^{+} \mathrm{CD} 25^{+}$Foxp $3^{+}$Tregs in the spleens and MLNs of mice in the TS+CR group mice was decreased $(P<0.05)$, showing that infection with $C$. rodentium could inhibit the expression of Tregs induced by T. spiralis (Fig. 6,7).

\section{Discussion}

2,4,6-Trinitrobenzene sulfonic acid (TNBS) and dinitrobenzenesulfonate (DNBS) mouse models are earlystage IBD animal models [18]. The damage caused in these models is highly concentrated, the course of the disease is acute, and the onset is short. Rat and mouse models of intestinal inflammation induced by the oral administration of dextran sulfate sodium (DSS) are also used. The DSS animal model is primarily an injury model, which is primarily used to study the influencing factors of promoting and inhibiting intestinal epithelial healing $[19,20]$. In the present study, mice were administered $C$. rodentium to induce IBD animal models. C. rodentium is an adhesion and shedding pathogen of the family Enterobacteriaceae to which gerbils and mice are susceptible, whereas hamsters and rats are not. This model has been widely used to study the immunoprotective role of $E$. coli and the mechanism of the host immune response against intestinal pathogens. C. rodentium -induced animal model mice showed obvious inflammatory symptoms in the colon, and the pathological characteristics were consistent with the IBD animal model.

Compared with the TS group, the TS+CR group showed a higher survival rate, reduced intestinal damage, decreased DAI score, and significantly reduced symptoms of intestinal inflammation, indicating that $T$. spiralis infection could alleviate colitis caused by $C$. rodentium. This finding is consistent with that observed in other worm infection studies [21, 22].

To better understand how worms suppress the occurrence of immunomodulatory diseases, recent research has focused on the interaction between the Th2- and Th1-type immune responses [23]. Th1 cells primarily secrete IL-2, IFN- $\gamma$, IFN- $\alpha$, and TNF- $\beta$, while Th2 cells primarily release IL-4, IL-5, IL-6 and IL-10 [24]. IL-12 plays an important role in Th1 cell-mediated immune response and inflammatory diseases [25]. IL-17 is a pro-inflammatory cytokine produced by $\mathrm{T}$ cells and is an early initiating factor of inflammatory responses induced by $\mathrm{T}$ cells [26]. In this study, IFN- $\gamma$ and IL-4, IL-10 were selected as representatives of Th1- and Th2-type cytokines. The number of Th cells was assessed by flow cytometry, and the results showed that $T$. spiralis infection could reduce the expression of IFN- $\gamma$, IL-17 and IL-12 and increase that of IL-4 and IL-10 in colitis mice. These results showed that T. spiralis infection causes a colitis mucosal immune response to switch from a Th1-type response to a protective Th2-type response. ELISA results showed changes in the levels of IgG1 and IgG2a in mouse serum, indicating that the levels of Th2-type cytokines caused by T. spiralis infection have an inhibitory effect on C. rodentium -induced Th1 type inflammation. These reactions reduce the intensity of the Th1-type immune response, thereby alleviating intestinal inflammation, and these results are consistent with the expected experimental results. From another perspective, colitis was also shown to stimulate the switch from a Th2-type immune response induced by T. spiralis larvae infection into a Th1-type immune response, reduce the intensity of the Th2-type immune response, and possibly alleviate the pathological changes attributed to the parasite infection in the body. T. spiralis and $C$. rodentium interact together to maintain immune balance in mice.

Tregs have a wide range of immunosuppressive effects. By producing regulatory cytokines, these cells maintain the balance of the immune system and prevent the development of autoimmune diseases [27]. Foxp3 is a marker molecule for $\mathrm{CD} 4{ }^{+} \mathrm{CD} 25^{+}$Tregs [28]. The results showed that compared with that observed in the control group, the number of $\mathrm{CD} 4^{+} \mathrm{CD} 25^{+} \mathrm{Foxp} 3^{+}$Tregs in the spleens and MLNs of mice in the TS group was increased, showing that T. spiralis infection could induce CD $4^{+} \mathrm{CD} 25^{+} \mathrm{Foxp} 3^{+}$Tregs production in mice In contrast, compared with that observed in the TS group, the number of $\mathrm{CD} 4^{+} \mathrm{CD} 25^{+} \mathrm{Foxp} 3^{+}$Tregs in the spleens and MLNs of mice in the TS+CR group were decreased, showing that $C$. rodentium infection can 
affect Tregs in the immune response induced by T. spiralis . The results of the present study are consistent with those of a previous study of worm infections that showed increased levels of Foxp $3^{+}$Tregs in mouse MLNs and intestinal mucosa lamina propria, inhibiting the mouse colon inflammatory response, as reported by Maloy et al [29].

In summary, the results of the present successfully showed that the effect of $T$. spiralis intervention on $C$. rodentium -induced colitis in mice is achieved by regulating the Th1/Th2 type immune response balance of the host.

\section{Acknowledgments}

We are grateful for the C. rodentium provided by Dr. Haining Shi Harvard Medical School, Boston, MA, USA. This work was supported by the National Key Research and Development Program of China (2017YFD0500400, 2017YFD0501200, 2017YFD0501000), the National Natural Science Foundation of China (31672528, 31941018) and Science and Technology Development Program of Jilin Province (20180201040NY, 20190301042NY).

\section{Conflict of Interest}

The authors declare that they have no conflict of interest.

\section{References}

1. Cho JH. The genetics and immunopathogenesis of inflammatory bowel disease. Nat Rev Immunol 2008;8 : 458-66.

2. Kassam Z, Lee CH, Yuan YH et al . Fecal Microbiota Transplantation for Clostridium difficileInfection: Systematic Review and Meta-Analysis. Am J Gastroenterol 2013;108 : 500-8.

3. Ballal SA, Veiga P, Fenn K et al . Host lysozyme-mediated lysis of Lactococcus lactisfacilitates delivery of colitis-attenuating superoxide dismutase to inflamed colons. P Natl Acad Sci USA 2015; 112 : 7803-8.

4. Collins JW, Keeney KM, Crepin VEet al . Citrobacter rodentium : infection, inflammation and the microbiota. Nat Rev Microbiol 2014; $12: 612-23$.

5. Xu M, Duan XY, Chen QY et al . Effect of compound sophorae decoction on dextran sodium sulfate (DSS)-induced colitis in mice by regulating Th17/Treg cell balance. Biomed Pharmacother 2019; 109 : 2396-408.

6. Park JS, Choi J, Jhun J et al . Lactobacillus acidophilus Improves Intestinal Inflammation in an Acute Colitis Mouse Model by Regulation of Th17 and Treg Cell Balance and Fibrosis Development. J Med Food 2018; 21 : 215-24.

7. Mundy R, MacDonald TT, Dougan Get al . Citrobacter rodentium of mice and man. Cell Microbiol 2005; $7: 1697-706$.

8. Symonds EL, Riedel CU, O'Mahony Det al. Involvement of T helper type 17 and regulatory T cell activity in Citrobacter rodentium invasion and inflammatory damage. Clin Exp Immunol 2009; 157 : 148-54.

9. Borody T, Torres M, Campbell Jet al . Reversal of Inflammatory Bowel Disease (IBD) with Recurrent Faecal Microbiota Transplants (FMT). Am J Gastroenterol 2011;106 : S366-S.

10. Lv Q, Wang K, Qiao SM et al . Norisoboldine, a natural AhR agonist, promotes Treg differentiation and attenuates colitis via targeting glycolysis and subsequent NAD(+)/SIRT1/SUV39H1/H3K9me3 signaling pathway. Cell Death Dis 2018;9 .

11. Gionchetti P, Calabrese C, Lauri A et al . The therapeutic potential of antibiotics and probiotics in the treatment of pouchitis. Expert Rev Gastroent 2015; 9 : 1175-81. 
12. Kanai T, Mikami Y, Hayashi A. A breakthrough in probiotics: Clostridium butyricum regulates gut homeostasis and anti-inflammatory response in inflammatory bowel disease. J Gastroenterol 2015; 50 : 928-39.

13. Lei H, Schmidt-Bleek K, Dienelt Aet al . Regulatory T cell-mediated anti-inflammatory effects promote successful tissue repair in both indirect and direct manners. Front Pharmacol 2015; 6 .

14. Yi Q, Wang JX, Song YX et al . Ascl2 facilitates IL-10 production in Th17 cells to restrain their pathogenicity in inflammatory bowel disease. Biochem Bioph Res Co 2019;510 : 435-41.

15. Jiang $\mathrm{Y}$, Yang G, Meng $\mathrm{F}$ et al . Immunological mechanisms involved in probiotic-mediated protection against Citrobacter rodentium -induced colitis. Benef Microbes 2016; 7 : 397-407.

16. Morris GP, Beck PL, Herridge MSet al . Hapten-induced model of chronic inflammation and ulceration in the rat colon. Gastroenterology 1989; 96 : 795-803.

17. Wallace JL, Keenan CM. An orally active inhibitor of leukotriene synthesis accelerates healing in a rat model of colitis. The American journal of physiology 1990; 258 : G527-34.

18. Ray A, Dittel BN. Interrelatedness between dysbiosis in the gut microbiota due to immunodeficiency and disease penetrance of colitis. Immunology 2015;146 : 359-68.

19. Olguin JE, Medina-Andrade I, Molina E, et al. Early and Partial Reduction in CD4(+)Foxp3(+) Regulatory T Cells during Colitis-Associated Colon Cancer Induces CD4(+) and CD8(+) T Cell Activation Inhibiting Tumorigenesis. J Cancer 2018;9 : 239-49.

20. Strisciuglio C, Miele E, Giugliano FP et al . Bifidobacteria Enhance Antigen Sampling and Processing by Dendritic Cells in Pediatric Inflammatory Bowel Disease. Inflamm Bowel Dis 2015; 21 : 1491-8.

21. Kosanovic M, Cvetkovic J, Gruden-Movsesijan A et al . Trichinella spiralis muscle larvae release extracellular vesicles with immunomodulatory properties. Parasite Immunol 2019; 41 .

22. Sun ZM, He ZH, Ma J. Separation of RORgammat function in Thymocyte development and Th17 differentiation. J Immunol 2018; 200 .

23. Yang Y, Bai X, Li CY et al . Molecular Characterization of Fructose-1,6-bisphosphate Aldolase From Trichinella spiralis and Its Potential in Inducing Immune Protection. Front Cell Infect Mi 2019; 9 .

24. Jin XM, Yang Y, Liu XL et al . Glutathione-S-transferase of Trichinella spiralis regulates maturation and function of dendritic cells. Parasitology 2019;146 : 1725-32.

25. Ren HN, Liu RD, Song YY et al . Label-free quantitative proteomic analysis of molting-related proteins of Trichinella spiralis intestinal infective larvae. Vet Res 2019; 50 .

26. Kang SA, Park MK, Park SKet al . Adoptive transfer of Trichinella spiralis -activated macrophages can ameliorate both Th1-and Th2-activated inflammation in murine models. Sci Rep-Uk 2019; 9 .

27. Song YN, Xu J, Wang XL et al . Regulation of host immune cells and cytokine production induced by Trichinella spiralis infection. Parasite 2019; 26 .

28. Xu JY, Liu MX, Yu PC et al . Effect of recombinant Trichinella spiralis cysteine proteinase inhibitor on TNBS-induced experimental inflammatory bowel disease in mice. Int Immunopharmacol 2019; 66 : 28-40.

29. Maloy KJ, Powrie F. Intestinal homeostasis and its breakdown in inflammatory bowel disease. Nature 2011; $474:$ 298-306.

Figure legends

Fig. 1 Changes in the weights of the mice in each group. The data are presented as the means $\pm \mathrm{SD}$ of 3 mice per group. 
Fig. 2 Changes in C. rodentium levels in faecal pellets. The data are presented as the means $\pm \mathrm{SD}$ of 3 mice per group.

Fig. 3 DAI assessment showing a lower value in the TS+CR group than that observed in the TS group. The data are presented as the means $\pm \mathrm{SD}$ of 3 mice per group.

Fig. 4 Histopathological analysis of the colons of mice. Colon samples were excised, fixed in $4 \%$ paraformaldehyde and embedded in paraffin. Sections were stained with H\&E for light microscopy assessments of epithelial damage (original magnification, $100 \times$ ).

A, Mice were treated with PBS as control, the structure of the colon villus was integrated, and the gland was clearly visible.

B, In mice from the CR group, intestinal epithelial cells were necrotic and shedding, intestinal villi were shortened and fused, and the crypts were extended. In addition, a large number of lymphocytes, neutrophils, and eosinophils had infiltrated into the mucosa and submucosa.

C, In mice from the TS group, a high number of goblet cells were detected, but there was no obvious pathological damage.

D, Hyperplasia of colonic epithelial tissue in the TS+CR mice disappeared, the lesions were limited to the mucosa, and the lamina propria of the mucosa was scattered with inflammatory cells.

Fig. 5 Changes in the IgG1 and IgG2a contents of serum post $C$. rodentium infection. The data are presented as the means $\pm \mathrm{SD}$ of 3 mice per group. ${ }^{*} P<0.05,{ }^{* *} P<0.01$, ${ }^{* * *} P<0.001$.

Fig. 6 Demonstration of the gating strategy for the flow cytometry analysis of mouse $\mathrm{CD} 4^{+} \mathrm{CD} 25^{+}$Foxp $3^{+}$Tregs and Th cells from spleens and MLNs 7 (A) and 14 days (B) after administration of $C$. rodentium. In this experiment single-cell suspensions were prepared from the spleens and MLNs of mice in each group and stained following surface and intracellular staining protocols. The data were collected with a FACSDiva flow cytometer and analysed. Lymphocytes were identified by their scatter properties (FSC-A x SSC-A plot).

Fig. 7 The number of Th cells in the spleens and MLNs of mice from the four groups at 7 and 14 days. Analysis of $\mathrm{CD}^{+} \mathrm{CD} 25^{+} \mathrm{Foxp} 3^{+}$Tregs in the spleens and MLNs of mice from each group. The number of $\mathrm{CD} 4{ }^{+} \mathrm{CD} 25^{+}$Foxp $^{+}{ }^{+}$Tregs in mice from the TS+CR group increased compared with that observed in the CR group. The data are presented as the means $\pm \mathrm{SD}$ of 3 mice per group. ${ }^{*} P<0.05,{ }^{* *} P<0.01,{ }^{* * *} P<$ 0.001 .

\section{Tables}

Table 1. Disease activity index score parameters.

\section{Hosted file}

Figure.docx available at https://authorea.com/users/336174/articles/472540-effect-oftrichinella-spiralis-intervention-on-citrobacter-rodentium-induced-experimental-colitisin-mice

\section{Hosted file}

Table.docx available at https://authorea.com/users/336174/articles/472540-effect-oftrichinella-spiralis-intervention-on-citrobacter-rodentium-induced-experimental-colitisin-mice

\section{Hosted file}

Cover letter.docx available at https://authorea.com/users/336174/articles/472540-effect-oftrichinella-spiralis-intervention-on-citrobacter-rodentium-induced-experimental-colitisin-mice 